\title{
HIGH FREQUENCY WAVES NEAR CUSP CAUSTICS
}

\author{
$\mathrm{BY}$ \\ E. KALLIGIANNAKI (Department of Mathematics, Univ. Crete, Heraklion, Crete, Greece, and \\ Institute of Applied and Computational Mathematics, FORTH, 71110 Heraklion, Crete, Greece), \\ TH. KATSAOUNIS (Department of Applied Mathematics, Univ. Crete, Heraklion, Crete, Greece, \\ and Institute of Applied and Computational Mathematics, FORTH, 71110 Heraklion, Crete, \\ Greece), \\ AND \\ G. N. MAKRAKIS (Institute of Applied and Computational Mathematics, FORTH, 71110 \\ Heraklion, Crete, Greece, and Department of Applied Mathematics, Univ. Crete, Heraklion, Crete, \\ Greece)
}

\begin{abstract}
It is well known that the usual harmonic ansatz of geometrical optics fails near caustics. However, uniform expansions exist which are valid near and on the caustics, and reduce asymptotically to the usual geometric field far enough from them. In this paper, we apply the Kravtsov-Ludwig technique for computing high-frequency fields near cusp caustics. We compare these fields, with those predicted by the geometrical optics, for a couple of model problems: first, the cusp generated by the evolution of a parabolic initial front in a homogeneous medium, a problem which arises in the highfrequency treatment of cylindrical aberrations, and second, the cusp formed by refraction of the rays emitted from a point source in a stratified medium with a weak interface. It turns out that inside and near the cusp, the geometrical optics solution is significantly different than the Kravtsov-Ludwig solution, but far enough from the caustic that the two solutions are, in fact, in very good agreement.
\end{abstract}

1. Introduction. The method of geometrical optics has been traditionally applied for investigating quantitatively high-frequency waves in inhomogeneous media. However, geometrical optics predicts infinite wave amplitudes on caustics and focal points, and zero fields in shadow regions (i.e., regions devoid of rays). Formation of caustics is a typical situation in optics, underwater acoustics, and seismology, as a result of multipath propagation from localized sources. Even in the simplest oceanic models and geophysical structures (see, e.g., Tolstoy and Clay [TC], Chap. 5, and Cěrvenỳ et. al. [CMP], Chap. 3 , respectively), a number of caustics occur, depending upon the position of the source and the stratification of the wave velocities. A recent review about the caustic formation, 
the related multivaluedness of the phase function, and the methods proposed over the years for constructing uniform high-frequency asymptotic solution of the Helmholtz and wave equations can be found in [KKM], [IKM].

Uniform caustic asymptotics have been widely used for acoustical and seismological computations (see, e.g., [CH1], [CH2], [HW], [BT] and the references cited therein). However, the limits of applicability of uniform asymptotic expansions have not been completely defined yet, as it has been recently pointed out by Asatryan and Kravtsov [ASK] who attempted to give a qualitative answer. Especially for stratified media with interfaces, where very complicated caustics may appear (see various examples in [KO2]), it turns out to be quite difficult to estimate analytically such limits. On the other hand, the quantitative answer requires a systematic numerical comparison between uniform asymptotic and geometrical optics solutions. In this paper, we perform such computations by applying the Kravtsov-Ludwig technique for the case of cusp caustics arising in a couple of typical configurations of ocean acoustics.

2. Classical geometrical optics. We consider the propagation of $n$-dimensional $(n=2,3)$ time-harmonic acoustic waves of frequency $\omega$ in a medium with variable refraction index $\eta(\mathbf{x})=c_{0} / c(\mathbf{x}), c_{0}$ being some reference sound velocity and $c(\mathbf{x})$ the sound velocity at the point $\mathbf{x}=\left(x_{1}, \ldots, x_{n}\right) \in M$, where $M$ is some unbounded domain in $\mathbb{R}_{\mathbf{x}}^{n}$. For the moment, we assume that $\eta \in C^{\infty}\left(\mathbb{R}_{\mathbf{x}}^{n}\right)$ and $\eta>0$. The wave field $u(\mathbf{x}, k)$ is governed by the Helmholtz equation

$$
\Delta u+k^{2} \eta^{2}(\mathbf{x}) u(\mathbf{x}, k)=f(\mathbf{x}), \quad \mathbf{x} \in M
$$

where $k=\omega / c_{0}$ is the wavenumber and $f$ is a compactly supported source generating the waves.

We are interested in the asymptotic behavior of $u(\mathbf{x}, k)$ as $k \rightarrow \infty$ (i.e., for very large frequencies $\omega$ ), assuming that $\mathbf{x}$ is confined in some compact subset $D$ of $M$ outside of the support of the source. The asymptotic decomposition of scattering solutions when $|\mathbf{x}|$ and $k$ simultaneously go to infinity is a rather complicated problem, since the caustics might go out to infinity. This problem has been studied rigorously for the scattering of a plane wave by a compact inhomogeneity in [V1], and for the case of a point source in $[\mathrm{KU}]$, under certain conditions of decay for $\eta(\mathbf{x})$ at infinity.

For fixed $k>0$ there is, in general, an infinite set of solutions of $(2.1)$, and we need a radiation condition to guarantee uniqueness (see, e.g., [CK] for scattering by compact inhomogeneities and [WED] for scattering by stratified media). This condition is essentially equivalent to the assumption that there is no energy flow from infinity, which in geometrical optics is translated to the condition that the rays must go out to infinity.

According to the WKB technique, we look for a formal asymptotic solution (FAS) of (2.1), that is, an expansion of the form [V2]

$$
u_{N}(\mathbf{x}, k)=e^{i k \Phi(\mathbf{x})} \sum_{l=0}^{N}(i k)^{-l} A_{l}(\mathbf{x}),
$$


the phase $\Phi$ and the amplitudes $A_{l}$ being real-valued functions in $C^{\infty}\left(\mathbb{R}_{\mathbf{x}}^{n}\right)$, which satisfies the asymptotic equation

$$
\left(\Delta+k^{2} n^{2}(\mathbf{x})\right) u_{N}(\mathbf{x}, k)=O\left(k^{-N_{1}}\right), \quad x \in D, \quad k \rightarrow \infty,
$$

where $N_{1} \rightarrow \infty$ as $N \rightarrow \infty$.

Substituting (2.2) into (2.3), and separating the powers $(i k)^{-l}, l=0,1, \ldots$, we obtain the eikonal equation

$$
(\nabla \Phi(\mathbf{x}))^{2}=\eta^{2}(\mathbf{x})
$$

for the phase function, and the following hierarchy of transport equations for the amplitudes:

$$
\begin{aligned}
2 \nabla \Phi \cdot \nabla A_{0}+\Delta \Phi(\mathbf{x}) A_{0}(\mathbf{x}) & =0 \\
2 \nabla \Phi \cdot \nabla A_{l}+\Delta \Phi(\mathbf{x}) A_{l}(\mathbf{x}) & =-\Delta A_{l-1}(\mathbf{x}), l=1,2, \ldots
\end{aligned}
$$

A standard way for solving the eikonal equation (2.4) is the method of bicharacteristics (see, e.g., [HO], Vol. I, Chap. VIII). Let $H(\mathbf{x}, \mathbf{p})$ be the Hamiltonian function

$$
H(\mathbf{x}, \mathbf{p})=\frac{1}{2}\left(|\mathbf{p}|^{2}-\eta^{2}(\mathbf{x})\right), \quad \mathbf{x} \in M, \mathbf{p} \in \mathbb{R}_{\mathbf{p}}^{n},
$$

corresponding to the Helmholtz equation (2.1), where $\mathbf{p}=\left(p_{1}, \ldots, p_{n}\right)$ is the momentum, conjugate to the position $\mathbf{x}=\left(x_{1}, \ldots, x_{n}\right)$. The associated Hamiltonian system reads as follows:

$$
\begin{aligned}
& \frac{d \mathbf{x}}{d t}=\nabla_{\mathbf{p}} H(\mathbf{x}, \mathbf{p})=\mathbf{p}, \\
& \frac{d \mathbf{p}}{d t}=-\nabla_{\mathbf{x}} H(\mathbf{x}, \mathbf{p})=\eta(\mathbf{x}) \nabla_{\mathbf{x}} \eta(\mathbf{x}) .
\end{aligned}
$$

Let $M_{0}=\left\{\mathbf{x}=\mathbf{x}^{0}(\theta), \theta=\left(\theta_{1}, \ldots, \theta_{n-1}\right) \in U_{0} \subset \mathbb{R}^{n-1}\right\}$ be an initial manifold in $\mathbb{R}_{\mathbf{x}}^{n}$. For $t=0$ we specify the initial conditions

$$
\mathbf{x}(0)=\mathbf{x}^{0}(\theta), \quad \mathbf{p}(0)=\mathbf{p}^{0}(\theta), \quad \theta \in U_{0}
$$

for the Hamiltonian system, and

$$
\Phi(\mathbf{x})=\Phi^{0}(\theta), \quad A_{l}(\mathbf{x})=A_{l}^{0}(\theta) \quad \text { for } \quad \mathbf{x}=\mathbf{x}^{0}(\theta) \in M_{0}
$$

for the eikonal and the transport equations. Note that the initial momentum $\mathbf{p}^{0}(\theta)$ must satisfy

$$
\left|\mathbf{p}^{0}(\theta)\right|^{2}=n^{2}\left(\mathbf{x}^{0}(\theta)\right), \quad \mathbf{x}_{0} \in M_{0} .
$$

$\mathbf{p}^{0}(\theta), \Phi^{0}(\theta)$, and $A_{l}^{0}(\theta)$ are given initial momentum, phase, and amplitude on the initial manifold $M_{0}$, which model the source function $f$ in (2.1). For this issue see [AK], [B] for the case of a point source and [KO2] for more general sources.

The trajectories $\Gamma(t, \theta)=\left\{\mathbf{x}=\mathbf{x}(t, \theta), \mathbf{p}=\mathbf{p}(t, \theta), t \geq 0, \theta \in U_{0}\right\}$ which solve the initial value problem (2.8), (2.9) in the phase space $\mathbb{R}_{\mathbf{x p}}^{2 n}$ are the bicharacteristics, and their projections $\gamma(t, \theta)=\left\{\mathbf{x}=\mathbf{x}(\tau, \theta), t \geq 0, \theta \in U_{0}\right\}$ onto $\mathbb{R}_{\mathbf{x}}^{n}$ are the rays of geometrical optics. 
We assume that $\mathbf{p}^{0}(\theta)$ is nowhere tangent to $M_{0}$, which means that the Cauchy problem for (2.4) is noncharacteristic. Then, the phase is given by

$$
\Phi(\mathbf{x}(t, \theta))=\Phi^{0}(\theta)+\int_{0}^{t} \mathbf{p}(\tau, \theta) \frac{d \mathbf{x}(\tau, \theta)}{d \tau} d \tau,
$$

where the integral is calculated along the bicharacteristics. The transformation

$$
(t, \theta) \mapsto \mathbf{x}(t, \theta)
$$

is one-to-one, provided that the Jacobian

$$
J(t, \theta)=\frac{\partial\left(x_{1}, \ldots, x_{n}\right)}{\partial\left(t, \theta_{1}, \ldots, \theta_{n-1}\right)}
$$

is non-zero. It is worth noticing that, even if $J \neq 0$ for $t=0$, it does not necessarily remain non-zero for all $t \geq 0$. Whenever $J$ vanishes, $(t, \theta)$ may be nonsmooth or multivalued functions of $\mathbf{x}$, and the rays $\gamma(t, \theta)$ may intersect, touch, and in general, have singularities. Therefore, the phase function $S=S(\mathbf{x})$ may be a multivalued or a nonsmooth function. However, the bicharacteristics $\Gamma(t, \theta)$ never intersect in the phase space, and this is the base for constructing uniform expansions near caustics.

The solution of the transport equation (2.5) for the principal amplitude $A_{0}$ along the rays is obtained by applying the divergence theorem in a ray tube, and it is given by

$$
A_{0}(\mathbf{x}(t, \theta))=\frac{\alpha_{0}(\theta)}{\sqrt{J(t, \theta)}},
$$

where $\alpha_{0}(\theta)=A_{0}^{0}(\theta)$ is the amplitude at the point $\mathbf{x}=\mathbf{x}^{0}(\theta)$ on the initial manifold.

The amplitude $A_{0}$ calculated by (2.14) blows up at the focal points $\{\mathbf{x}=\mathbf{x}(t, \theta)$ : $J(t, \theta)=0\}$. The manifold generated by the focal points, that is, the envelope of the family of the rays, is the caustic. Therefore, the WKB technique fails to predict the correct amplitudes on the caustic. The unbounded increase of the amplitude on the caustic is a consequence of the energy conservation and the diminishing of the ray tubes in the caustic vicinity. This phenomenon is related to the way of solving the problem, rather than with the structure of the wavefield itself, since we know that the solutions of the Helmholtz equation are analytic outside the support of singular sources or boundary data, whenever the refraction index is smooth. In fact, a boundary layer analysis [BK] shows that the ray structure breaks down near the caustic, and in a region adjacent to this layer, where the modal structure of the wave field is dominant, it is impossible to separate the waves approaching the caustic from those leaving it. Nevertheless, energy concentration in this region makes the caustic detectable, as the field is always finite but very strong there (cf. $[\mathrm{KR}])$.

3. Kravtsov-Ludwig method for the cusp. The construction of uniform highfrequency solutions of the Helmholtz equation (2.1) by the Kravtsov-Ludwig method is based on the integral representation (see, e.g., [KR], [LU], [DUI])

$$
u(\mathbf{x})=\left(\frac{i k}{2 \pi}\right)^{1 / 2} \int_{\Xi} e^{i k S(\mathbf{x}, \xi)} a(\mathbf{x}, \xi) d \xi, \quad \xi \in \Xi \subset \mathbb{R}_{\xi},
$$


instead of the FAS (2.2). Here $S$ and $a$ satisfy, identically with respect to $\xi$, the eikonal equation (2.4) and the transport equation (2.5), respectively. The integral (3.1) can be regarded as a continuous superposition of oscillatory functions of the form (2.2). The physical motivation underlying the method is the fact that in every small region in which the refraction index of the medium can be considered as constant and the wave front as plane, the field can be represented as a superposition of plane waves $a e^{i S}$, where $a$ and $\nabla S$ vary slowly in transition from one region to the next.

For large $k$, the stationary phase lemma (see, e.g., $[\mathrm{BH}]$, p. 219) says that the main contribution to the integral (3.1) comes from the stationary points $\xi_{j}(\mathbf{x})$, for which $\partial_{\xi} S\left(\mathbf{x}, \xi_{j}(\mathbf{x})\right)=0$. In the case of the usual (single phase) geometrical optics, we can take $S(\mathbf{x}, \xi)=\phi(\mathbf{x})-\xi^{2}$, which has only one simple stationary point $\xi=0$. Then the oscillatory integral (3.1) reduces asymptotically to $(2.2)$. When there are more than one simple stationary points $\xi_{j}(\mathbf{x})$, i.e.,

$$
\partial_{\xi} S\left(\mathbf{x}, \xi_{j}(\mathbf{x})\right)=0, \quad \partial_{\xi}^{2} S\left(\mathbf{x}, \xi_{j}(\mathbf{x})\right) \neq 0
$$

we obtain the asymptotic expansion

$$
u(\mathbf{x}) \sim \sum_{j} A_{0}^{j}(\mathbf{x}) e^{i k S_{j}(\mathbf{x})},
$$

where

$$
\begin{gathered}
S_{j}(\mathbf{x})=S\left(\mathbf{x}, \xi_{j}(\mathbf{x})\right) \\
A_{0}^{j}(\mathbf{x})=\exp \left(i\left(\frac{\pi}{2}+\operatorname{sgn}\left(\partial_{\xi}^{2} S\left(\mathbf{x}, \xi_{j}(\mathbf{x})\right)\right)\right)\right) \frac{a\left(\mathbf{x}, \xi_{j}(\mathbf{x})\right)}{\sqrt{\left|\partial_{\xi}^{2} S\left(\mathbf{x}, \xi_{j}(\mathbf{x})\right)\right|}}
\end{gathered}
$$

and the summation in (3.2) extends over all of the stationary points. The amplitudes $A_{0}^{j}(\mathbf{x})$ are solutions of the principal transport equation (2.5). The expansion (3.2) fails whenever $\partial_{\xi}^{2} S\left(\mathbf{x}, \xi_{j}(\mathbf{x})\right)=0$, that is, for the stationary points of higher multiplicity. The appearance of multiple stationary points is associated with the formation of caustics. In this case, modified stationary phase lemmas must be applied (see, e.g., [BO]).

Near caustics the phase is a multivalued function, which, in general, cannot be derived by integration along bicharacteristics (Eq. (2.11)). In this case, representation theorems for the phase function are derived by the methods of singularity theory (see, e.g., [AVH]), provided that the type of the caustic is known. For a cusp caustic it can be shown that the phase function and the amplitude must have the form ([GS], p. 441, Proposition 7.1 for $k=4$, and $[\mathrm{KR}])$

$$
S(\mathbf{x}, \xi)=\phi(\mathbf{x})+\rho_{1}(\mathbf{x}) \xi-\rho_{2}(\mathbf{x}) \frac{\xi^{2}}{2}+\frac{\xi^{4}}{4}
$$

and

$$
a(\mathbf{x}, \xi)=g_{0}(\mathbf{x})+\xi g_{1}(\mathbf{x})+\xi^{2} g_{2}(\mathbf{x})+f(\mathbf{x}, \xi) \partial_{\xi} S(\mathbf{x}, \xi),
$$

$f(\mathbf{x}, \xi)$ being a smooth function.

The stationary points $\xi_{j}(\mathbf{x})$ are the roots of the cubic equation

$$
\partial_{\xi} S(\mathbf{x}, \xi)=\rho_{1}(\mathbf{x})-\rho_{2}(\mathbf{x}) \xi+\xi^{3}=0
$$




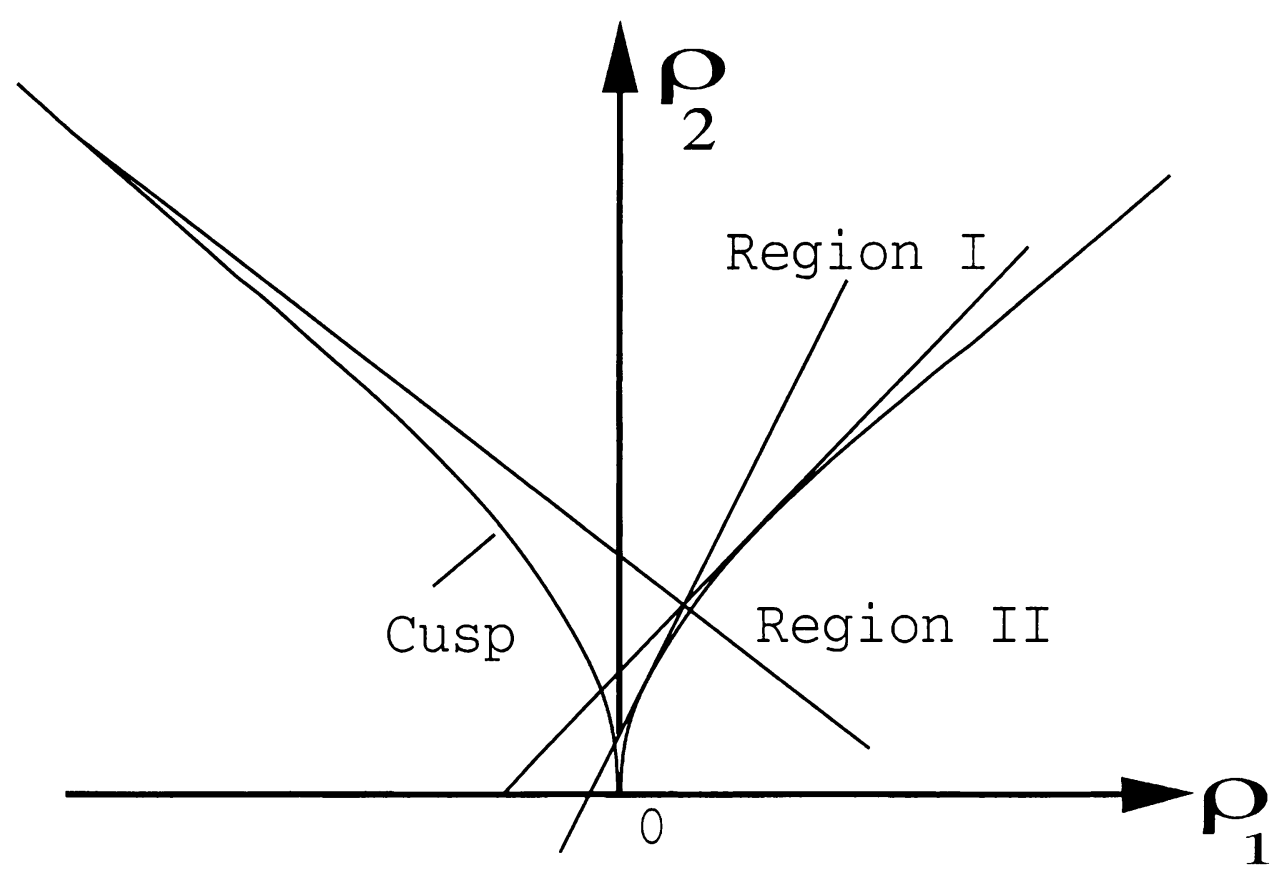

FIG. 3.1. The geometry of a cusp caustic

and they are given by the formulae

$$
\begin{aligned}
& \xi_{1}\left(\rho_{1}, \rho_{2}\right)=A+B \\
& \xi_{2}\left(\rho_{1}, \rho_{2}\right)=-\frac{1}{2}(A+B)+i \frac{\sqrt{3}}{2}(A-B), \\
& \xi_{3}\left(\rho_{1}, \rho_{2}\right)=-\frac{1}{2}(A+B)-i \frac{\sqrt{3}}{2}(A-B),
\end{aligned}
$$

where

$$
A=\left(\frac{\rho_{1}}{2}+\sqrt{D}\right)^{1 / 3}, \quad B=\left(\frac{\rho_{1}}{2}-\sqrt{D}\right)^{1 / 3}, \quad D=\frac{\rho_{1}^{2}}{4}-\frac{\rho_{2}^{3}}{27} .
$$

Note that $D=0$ at the points of the cusp (see Fig. 3.1):

$$
\rho_{1}^{2}=\frac{4 \rho_{2}^{3}}{27}
$$

From $(3.7 \mathrm{a})$ and $(3.7 \mathrm{c})$ it follows that there are three real stationary points in Region I, a double one on the sides of the cusp, and a triple one, equal to zero, at the "beak" of the cusp, while there is only one real stationary point in Region II (single-phase region).

It is worth noticing that each real stationary point corresponds to a real, and therefore there are three rays passing through any point inside the cusp (Region I), but only one ray through each point outside the cusp (Region II). At the sides of the cusp the double stationary point corresponds to a pair of coalescing rays tangent to the caustic, while the third one corresponds to the ray which crosses the caustic transversely. Finally, at the 
"beak" of the cusp (3.8), the triple stationary point $\xi_{1}=\xi_{2}=\xi_{3}=0$ corresponds to the three rays coalescing at this point $\rho_{1}=\rho_{2}=0$.

According to the matching principle $[\mathrm{LU}],[\mathrm{KR}]$, we apply the stationary phase lemma for the integral (3.1) with phase (3.4) and amplitude (3.5) (see, e.g., [BH], p. 221, [WO], Chap. VII), and we match the obtained amplitudes and phases with the geometrical ones. From the matching of the amplitudes we obtain the system

$$
\begin{gathered}
g_{0}(\mathbf{x})+\xi_{j} g_{1}(\mathbf{x})+\xi_{j}^{2} g_{2}(\mathbf{x})=A_{j}(\mathbf{x})\left(\partial_{\xi}^{2} S\left(\mathbf{x}, \xi_{j}(\mathbf{x})\right)\right)^{1 / 2} \\
=A_{j}(\mathbf{x})\left(3 \xi_{j}^{2}(\mathbf{x})-\rho_{2}(\mathbf{x})\right)^{1 / 2}, \quad j=1,2,3,
\end{gathered}
$$

where $A_{j}(\mathbf{x})$ are the geometrical amplitudes given by $(2.14)$,

$$
A_{j}(\mathbf{x})=\frac{\alpha_{0}\left(\theta_{j}\right)}{\sqrt{J_{j}(\mathbf{x})}} .
$$

Here $\theta_{j}$ are the values of the parameter at the initial manifold corresponding to the $j$ th ray, while $\alpha_{0}\left(\theta_{j}\right)$ is the corresponding initial amplitude and $J_{j}(\mathbf{x})$ is the value of the Jacobian calculated along the $j$ th ray (see Eq. (2.14)).

From the matching of the phases $\widetilde{S}_{j}\left(\phi, \rho_{1}, \rho_{2}\right)=S\left(\mathbf{x}, \xi_{j}\left(\rho_{1}, \rho_{2}\right)\right)$ with the geometrical phases $\Phi_{j}(\mathbf{x})$ which are computed by integration along the $j$ th ray $(j=1,2,3)$ (Eq. $(2.11)$ ), we obtain

$$
\widetilde{S}\left(\phi, \rho_{1}, \rho_{2}\right)=\phi(\mathbf{x})+\rho_{1} \xi_{j}\left(\rho_{1}, \rho_{2}\right)-\rho_{2} \frac{\xi_{j}^{2}\left(\rho_{1}, \rho_{2}\right)}{2}+\frac{\xi_{j}^{4}\left(\rho_{1}, \rho_{2}\right)}{4}=\Phi_{j}(\mathbf{x}), \quad j=1,2,3 .
$$

We solve first the nonlinear system (3.11) for $\rho_{1}(\mathbf{x}), \rho_{2}(\mathbf{x})$, and $\phi(\mathbf{x})$, and thereafter we calculate $\xi_{j}(\mathbf{x})=\xi_{j}\left(\rho_{1}(\mathbf{x}), \rho_{2}(\mathbf{x})\right)$ by the relations $(3.7 \mathrm{a})-(3.7 \mathrm{c})$. Then, we solve the linear system (3.9) for the modified amplitudes $g_{0}, g_{1}, g_{2}$. It can be shown using (3.10) and the system (3.9) that the modified amplitudes $g_{0}, g_{1}$, and $g_{2}$ remain finite on the caustic [LU].

Finally, using (3.1), (3.4), (3.5), and (3.12), we obtain the asymptotic expansion

$$
\begin{gathered}
u(\mathbf{x})=(2 \pi)^{-\frac{1}{2}} e^{i \frac{\pi}{4}} e^{i k \phi(\mathbf{x})}\left(g_{0}(\mathbf{x}) k^{\frac{1}{4}} Y_{0}(-a, b)-i k^{-\frac{1}{2}} g_{1}(\mathbf{x}) \partial_{b} Y_{0}(-a, b)\right. \\
\left.+2 i k^{-\frac{1}{2}} g_{2}(\mathbf{x}) \partial_{a} Y_{0}(-a, b)\right)+O\left(k^{-1}\right), \quad k \rightarrow \infty,
\end{gathered}
$$

where

$$
Y_{0}(-a, b)=\int_{\mathbb{R}} \exp \left(i\left(\frac{t^{4}}{4}-a \frac{t^{2}}{2}+b t\right)\right) d t
$$

is the Pearcey's integral [BO], [WO], and

$$
a=k^{\frac{1}{2}} \rho_{2} \quad \text { and } \quad b=k^{\frac{3}{4}} \rho_{1} .
$$

The integral (3.13) converges in the usual Lebesgue sense, i.e., without regularization [GS]. The three terms in the expansion (3.12) correspond to the first three terms in (3.5), since the stationary phase lemma gets rid of the last term in (3.5), the contribution of which is included in the remainder.

Obviously we can write

$$
b=\mu a^{\frac{3}{2}}, \quad \mu=\frac{\rho_{1}}{\rho_{2}^{3 / 2}}
$$


where $\mu$ is the uniformity parameter used in the uniform asymptotic expansion of $Y_{0}$ constructed by Kaminski [KAM]. Roughly speaking, it provides a measure of the distance of the point $\left(\rho_{1}, \rho_{2}\right)$ from the caustic (where $\mu$ takes the value $\mu_{c}=2 / \sqrt{2} 7$ ).

For the numerical computation of the Pearcey integral in the numerical examples of Sec. 4, we combined series expansion ([CF1], [CF2]) with numerical integration after deforming the integral (3.13) in the complex $t$-plane, and the above-mentioned uniform asymptotic expansion to achieve accurate results for the whole range of arguments $a, b$ [KAL]. The numerical-asymptotic computation of the high-frequency field by formula (3.12) relies on the accurate numerical solution of the nonlinear system (3.11). For solving this system, we rewrite it in the form

$$
\begin{gathered}
\phi=-\rho_{1} \xi_{1}+\rho_{2} \frac{\xi_{1}^{2}}{2}-\frac{\xi_{1}^{4}}{4}+\Phi_{1}, \\
\rho_{1}\left(\xi_{1}-\xi_{2}\right)-\frac{\rho_{2}}{2}\left(\xi_{1}^{2}-\xi_{2}^{2}\right)+\frac{1}{4}\left(\xi_{1}^{4}-\xi_{2}^{4}\right)=\Phi_{1}-\Phi_{2}, \\
\rho_{1}\left(\xi_{2}-\xi_{3}\right)-\frac{\rho_{2}}{2}\left(\xi_{2}^{2}-\xi_{3}^{2}\right)+\frac{1}{4}\left(\xi_{2}^{4}-\xi_{3}^{4}\right)=\Phi_{2}-\Phi_{3} .
\end{gathered}
$$

Using the formulae

$$
\xi_{1}+\xi_{2}+\xi_{3}=0, \quad \xi_{1} \xi_{2} \xi_{3}=-\rho_{1}, \quad \xi_{1} \xi_{2}+\xi_{2} \xi_{3}+\xi_{1} \xi_{3}=-\rho_{2}
$$

connecting the roots of the cubic equation (3.6) with $\rho_{1}, \rho_{2}$ we express the equations $(3.16 \mathrm{~b}),(3.16 \mathrm{c})$ as a system with respect to $\xi_{2}, \xi_{3}$ :

$$
\begin{aligned}
& F\left(\xi_{2}, \xi_{3}\right)=-\xi_{3}^{4} / 4-2 \xi_{2}^{3} \xi_{3}-3 \xi_{2} \xi_{3}^{3} / 2-3 \xi_{2}^{2} \xi_{3}^{2}=\Phi_{1}-\Phi_{2} \\
& H\left(\xi_{2}, \xi_{3}\right)=-\xi_{2}^{4} / 4-2 \xi_{2} \xi_{3}^{3} / 2+\xi_{2}^{3} \xi_{3} / 2+\xi_{3}^{4} / 4=\Phi_{2}-\Phi_{3}
\end{aligned}
$$

Note that the roots $\xi_{2}, \xi_{3}$ correspond to the rays which coalesce as we approach the caustic. The system (3.18a), (3.18b) is solved by Newton's method assuming as convergence criterion the absolute distance between successive approximations of the roots to be less than a given tolerance $\varepsilon$. In our numerical examples (Sec. 4), we take $\varepsilon=10^{-12}$. The initial values for starting Newton's algorithm are selected as the roots of the cubic equation (3.6) where the coordinates $\rho_{1}, \rho_{2}$ are found using the geometric transformation mapping the particular caustic to the standard form (3.8) (see Eqs. (4.8), (4.19a,b) and $(4.23 \mathrm{a}, \mathrm{b})$ below).

As we approach the beak of the cusp, the Jacobian of the system (3.18a), (3.18b) goes to zero together with $\xi_{2}, \xi_{3}$, and with the same order with respect to the distance from the beak. However, since $\left(\Phi_{2}-\Phi_{3}\right)$ goes also to zero and $\left(\Phi_{2}-\Phi_{3}\right) / H\left(\xi_{2}, \xi_{3}\right)$ remains bounded away from zero, the computation is stable up to distances of the order $10^{-10}$ from the beak. At such distances, the value of the uniformity parameter is $\mu=$ 0.3124999612154552 , while on the caustic it has the value $\mu=2 / \sqrt{27}=0.38490018 \ldots$ 


\section{Numerical examples.}

4.1. Evolution of parabolic front. The treatment of high-frequency, paraxial propagation from a point source in an inhomogeneous half-space $(\eta=1)$ leads, after certain transformations [KO1], to the computation of the high-frequency field generated by a propagating front, which is initially parabolic and of unit strength (cf. [ZD]). In the framework of geometrical optics, we consider the eikonal equation

$$
(\nabla \Phi(x, z))^{2}=1
$$

with initial data

$$
\left.\Phi\right|_{\Gamma}=0,\left.\quad \partial_{\nu} \Phi\right|_{\Gamma}=1
$$

given on the parabola (initial manifold)

$$
M_{0}=\left\{(x, z) \mid x=\frac{\zeta^{2}}{2 \sigma}, \quad z=\zeta, \zeta \in \mathbb{R}\right\} .
$$

The Hamiltonian $(2.7)$ is given by $H(\mathbf{x}, \mathbf{p})=\frac{1}{2}\left(|\mathbf{p}|^{2}-1\right)$. Solving the system $(2.8)$ for the above Hamiltonian, we find the rays

$$
\begin{aligned}
& x(\tau, \zeta)=\frac{\sigma}{\sqrt{\sigma^{2}+\zeta^{2}}} \tau+\frac{\zeta^{2}}{2 \sigma}, \\
& z(\tau, \zeta)=-\frac{\zeta}{\sqrt{\sigma^{2}+\zeta^{2}}} \tau+\zeta
\end{aligned}
$$

The Jacobian (2.13) is given by

$$
J(\tau, \zeta)=\frac{1}{\sigma} \sqrt{\sigma^{2}+\zeta^{2}}-\frac{\sigma}{\sigma^{2}+\zeta^{2}} \tau,
$$

and accordingly the caustic is the curve

$$
x=\sigma+\frac{3 \sigma^{\frac{1}{3}}}{2} z^{\frac{2}{3}} .
$$

Eliminating the parameter $\tau$ from the equations $(4.4 \mathrm{a}, \mathrm{b})$, we obtain the cubic equation

$$
\zeta^{3}+2 \sigma(\sigma-x) \zeta-2 \sigma^{2} z=0 .
$$

Note that by the simple change of coordinates

$$
\xi=\frac{\zeta}{\sqrt{2 \sigma}}, \quad \rho_{1}=-\sqrt{\frac{\sigma}{2}} z, \quad \rho_{2}=x-\sigma,
$$

we rewrite the equation of the caustic (4.6) in the form (3.8), while the cubic equation (4.7) takes the form (3.6). This transformation, as we have already mentioned in Sec. 3, enables us only to choose the initial values of $\xi_{2}, \xi_{3}$ when solving the system $(3.18 \mathrm{a}, \mathrm{b})$ by Newton's method. However, the correct Kravtsov-Ludwig coordinates $\rho_{1}, \rho_{2}$ to be inserted in the formula (3.13) have to be found from the solution of the nonlinear system (3.16).

For the computation of the modified amplitudes $g_{0}, g_{1}, g_{2}$ we proceed as we described in Sec. 3. The geometrical phases are derived by integration along the rays, and they are 


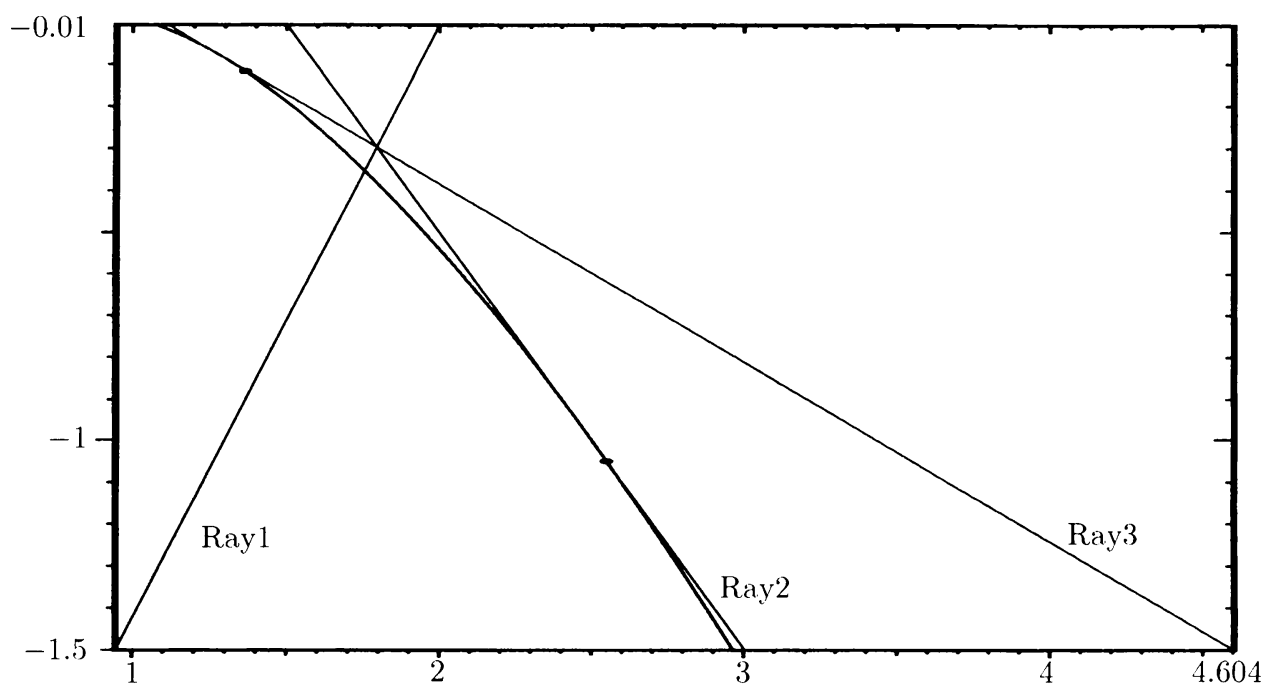

FIG. 4.1. Caustic and rays $(\sigma=1)$

given by

$$
\begin{aligned}
\Phi_{j}(x, z) & =\int_{0}^{\tau_{j}(x, y)} n^{2}\left(x\left(t, \zeta_{j}\right), z\left(t, \zeta_{j}\right)\right) d t \\
& =\tau_{j}(x, z)=\left(\zeta_{j}-z\right) \frac{\sqrt{\sigma^{2}+\zeta_{j}^{2}}}{\zeta_{j}}
\end{aligned}
$$

while the geometrical amplitudes are

$$
A_{j}(\mathbf{x})=\frac{\alpha_{0}\left(\zeta_{j}\right)}{\sqrt{J_{j}(\mathbf{x})}},
$$

where $J_{j}(\mathbf{x})=J\left(\tau_{j}, \zeta_{j}\right)$ are given by (4.5). In the sequel, for simplicity, we assume that $\alpha_{0}=1$ everywhere on $\Gamma$.

Recall that inside the cusp (Region $\mathrm{I}$ ), the rays which pass through the point $\mathbf{x}=(x, z)$ at the times $\tau_{j}=\tau_{j}(x, z), j=1,2,3$, originate at the points of the initial parabola $\Gamma$ corresponding to the values $\zeta_{j}=\zeta_{j}(x, z)=\xi_{j}\left(\rho_{1}, \rho_{2}\right) \sqrt{2 \sigma}$, where $\xi_{j}$ are given by (3.7a)(3.7c), with $\rho_{1}, \rho_{2}$ defined in (4.8).

In Figures $4.2 \mathrm{a}, 4.2 \mathrm{~b}$, and $4.2 \mathrm{c}$, we compare the amplitudes of the field predicted by geometrical optics (GO) with that predicted by the Kravtsov-Ludwig formula (KL) for relatively high frequencies $(k=100)$ along the three rays passing through the point $\left(x_{0}, z_{0}\right)=(1.7937,-0.382)$ inside the cusp (4.6) with $\sigma=1$ (Fig. 4.1). We compute the amplitudes as functions of $|z|$. The solid lines represent the GO solution which blows up along each ray as we approach the caustic. The dashed lines represent the KL solution when the Pearcey integral is computed using the uniform asymptotic expansion by Kaminski $[\mathrm{KAM}]$, while the dotted lines represent the KL solution when we compute the 


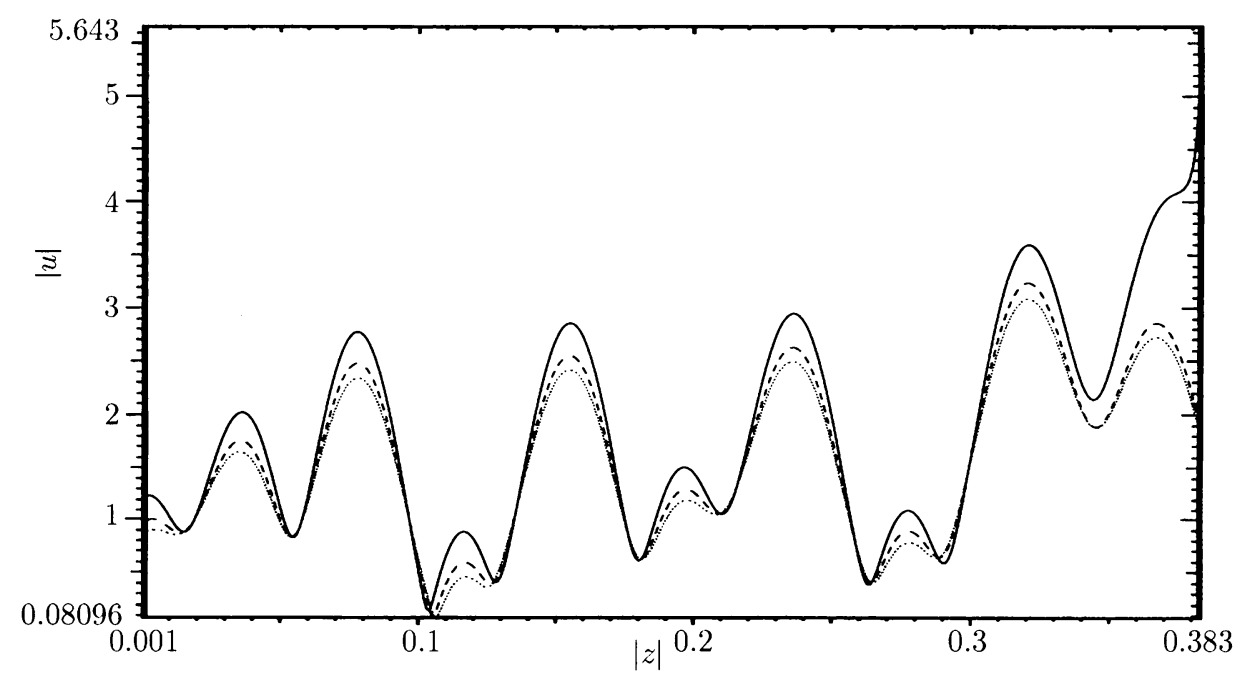

FIG. 4.2 a. $|u|$ vs. $|z|$ along ray 1

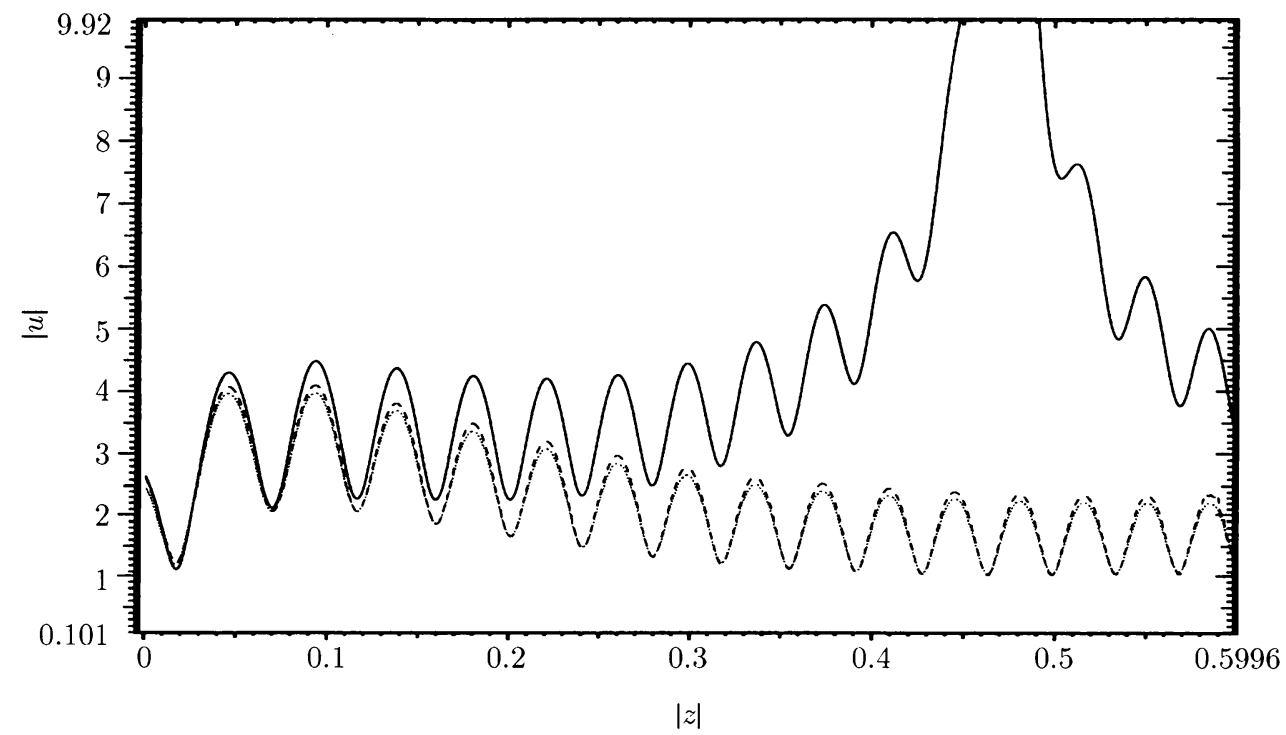

FIG. $4.2 \mathrm{~b} .|u|$ vs. $|z|$ along ray 2

Pearcey integral by direct numerical integration. In the latter case the numerical integration fails for very large $k|z|$. However, uniform asymptotics and numerical integration give almost identical results for a satisfactory range of $|z|$ for each $k$, thus providing some evidence that the computation is accurate for large values of $k|z|$. We observe that GO tends to match with the KL solution far away from the caustic. The distance from the caustic for satisfactory matching depends, in general, on the particular wave number $k$. 


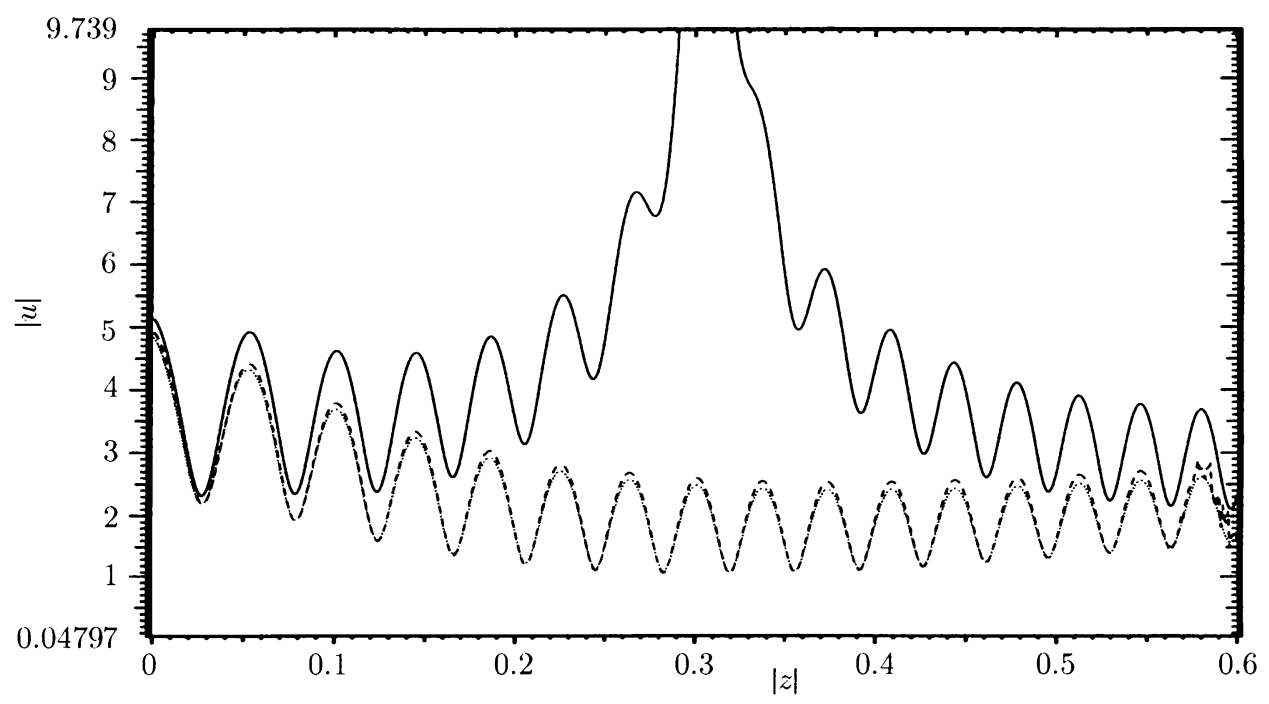

FIG. 4.2 c. $|u|$ vs. $|z|$ along ray 3

4.2. Point source a stratified medium. Now we consider the high-frequency field a point source at $\left(x_{0}, z_{0}\right)=\left(0, z_{0}\right), z_{0}<0$, in a medium with refraction index $(\alpha>0)$ :

$$
\eta^{2}(z)= \begin{cases}1, & z<0 \\ (1+\alpha z)^{-2}, & z>0\end{cases}
$$

The initial conditions (2.9) for the Hamiltonian system (2.8a), (2.8b), in this case are $\mathbf{x}_{0}=\left(0, z_{0}\right), \mathbf{p}_{0}=(\cos \theta, \sin \theta)$. The geometrical optics field of a point source has been studied using matched asymptotic expansions and boundary layer methods by Avila and Keller $[\mathrm{AK}]$ and Babich $[\mathrm{B}]$. A detailed discussion of the point source from the viewpoint of singularity theory is presented in [IKM]. A Hamiltonian ray-tracing algorithm by using adaptive Runge-Kutta solvers for solving the system (2.8a), (2.8b), and (2.9) when $\eta^{2}$ has discontinuous derivatives was developed in [KKM].

The rays shot from the source (direct rays) with initial angle $\theta, 0<\theta<\pi / 2$, are given by

$$
x(t, \theta)=t \cdot \cos \theta, \quad z(t, \theta)=z_{0}+t \cdot \sin \theta,
$$

i.e., they are straight lines in the homogeneous half space $z<0$. These rays hit the interface $z=0$ at $x_{u}(\theta)=-z_{0} c t g \theta$, and they are refracted upwards into the half space $z>0$. The upwards refracted rays are given by

$$
x(t, \theta)=t \cdot \cos \theta-z_{0} \operatorname{ctg} \theta, \quad z(t, \theta)=\frac{1}{\alpha}\left(\frac{\sqrt{1-\left(\alpha t \cos ^{2} \theta-\sin \theta\right)^{2}}}{\cos \theta}-1\right) .
$$

The rays (4.12), due to continuous refraction, return back to the interface $x_{d}(\theta)=$ $\frac{1}{\alpha}\left(2 \tan \theta-\alpha z_{0} \operatorname{ctg} \theta\right)$, and then they are refracted downwards into $z<0$. The downward 


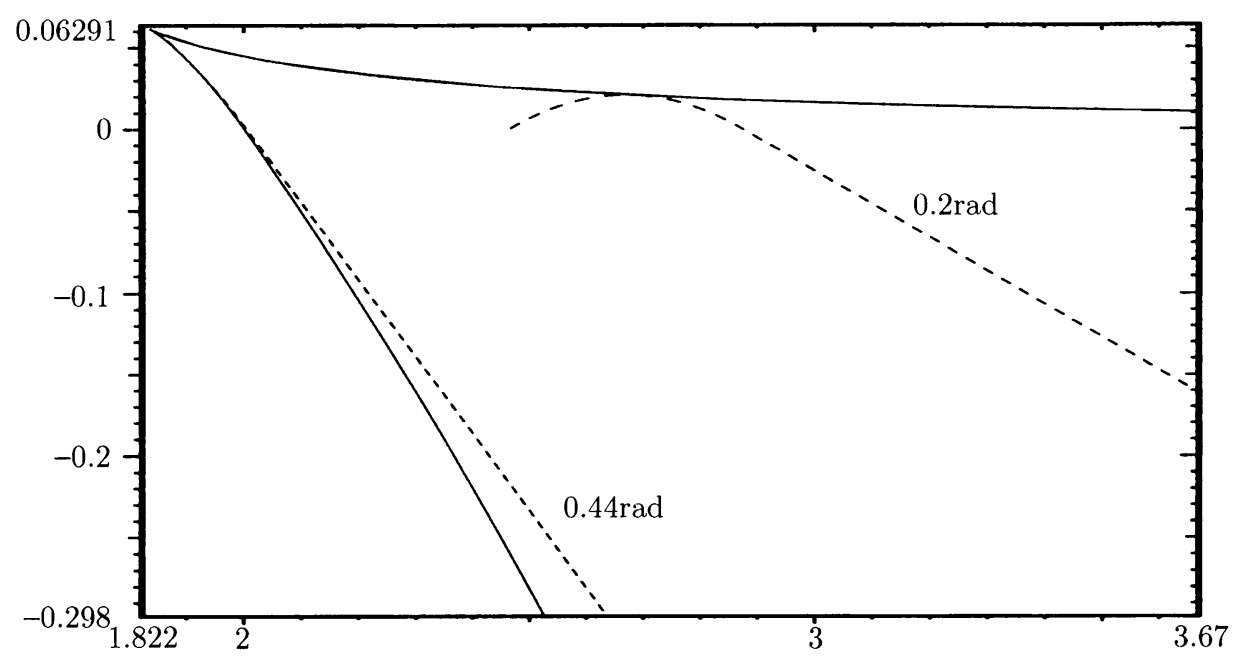

FIG. 4.3. Caustic and rays launched with $\theta=0.2 \mathrm{rad}$ and $\theta=0.44 \mathrm{rad}$

refracted rays in $z<0$ are given by

$$
x(t, \theta)=t \cdot \cos \theta+\frac{1}{\alpha}\left(2 \tan \theta-\alpha z_{0} \operatorname{ctg} \theta\right), \quad z(t, \theta)=-t \cdot \sin \theta .
$$

The Jacobian for the rays (4.12) is given by

$$
\begin{aligned}
J=( & \left(\alpha \sqrt{1-\left(\alpha t \cos ^{2} \theta-\sin \theta\right)^{2}}\right)^{-1} \\
& \left(1-\left(\alpha t \cos ^{2} \theta-\sin \theta\right)^{2} \tan \theta+\cos \theta\left(\alpha t \cos ^{2} \theta-\sin \theta\right)(2 \alpha t \sin \theta+1)(4.14 \mathrm{a})\right. \\
& \left.-\alpha \cos \theta\left(\alpha t \cos ^{2} \theta-\sin \theta\right)\left(t \sin \theta-\frac{z_{0}}{\sin ^{2} \theta}\right)\right)
\end{aligned}
$$

and for the rays (4.13) by

$$
J=-t+\frac{\sin \theta}{\alpha}\left(\frac{2}{\cos ^{2} \theta}+\frac{\alpha z_{0}}{\sin ^{2} \theta}\right) .
$$

After some cumbersome manipulations to eliminate $t$ between (4.12), (4.13a), (4.14a), and $(4.14 \mathrm{~b})$, we find that the caustic in the upper half space $z>0$ is given by the parametric equations

$$
\begin{aligned}
x_{c}(\theta) & =-z_{0} \operatorname{ctg} \theta \frac{\left(\alpha z_{0}\right) \cos ^{2} \theta}{\sin ^{2} \theta+\left(\alpha z_{0}\right) \cos ^{2} \theta} \\
\left(1+\alpha z_{c}(\theta)\right)^{2} & =\frac{1}{\cos ^{2} \theta}-\tan ^{2} \theta \frac{\sin ^{4} \theta}{\left(\sin ^{2} \theta+\left(\alpha z_{0}\right) \cos ^{2} \theta\right)^{2}}
\end{aligned}
$$

and in the lower half space $z<0$ by

$$
x_{c}(\theta)=\frac{4}{\alpha} \tan \theta, \quad z_{c}(\theta)=-\frac{1}{\alpha}\left(2 \tan ^{2} \theta+\alpha z_{0}\right) .
$$




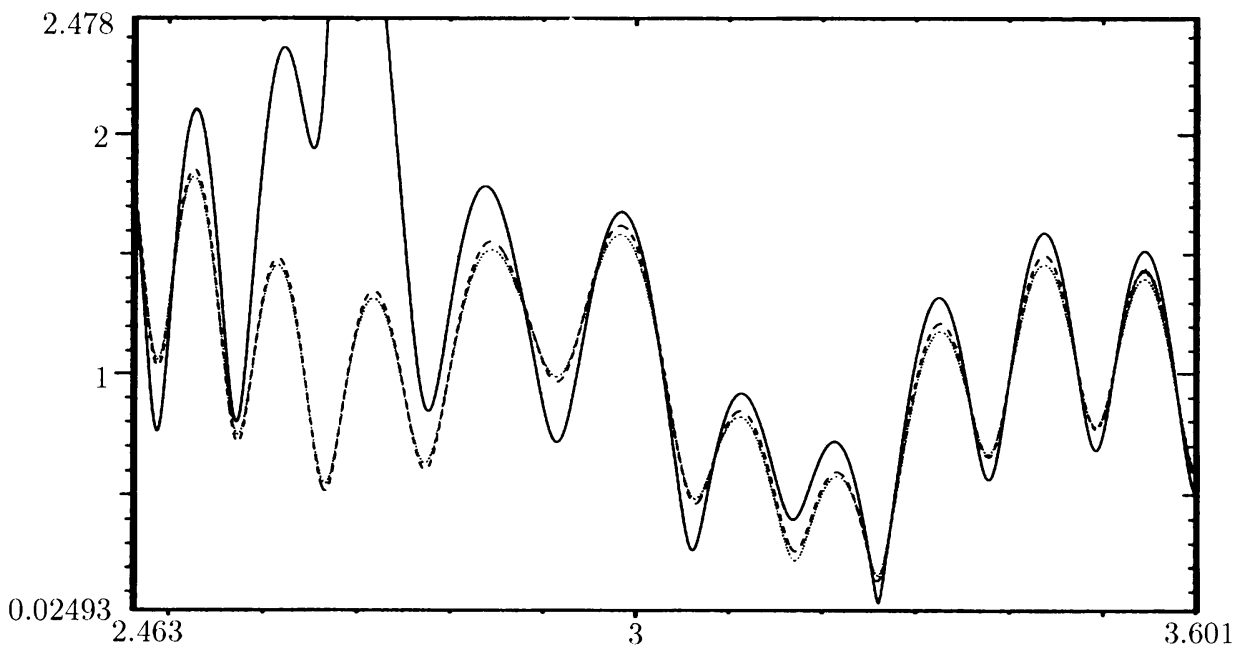

FiG. 4.4a. $|u|$ vs. $|x|, \theta=0.2 \mathrm{rad}, k=200$

The point

$$
x_{K}=\frac{3 \sqrt{3}}{2 \sqrt{-\left(\alpha z_{0}\right)}}\left(-z_{0}\right), \quad z_{K}=\frac{1}{\alpha}\left(-1+\sqrt{1-\frac{\alpha z_{0}}{4}}\right),
$$

given by (4.15a) for $\theta=\theta_{K}=\arccos \left(\sqrt{\frac{3}{3-\alpha z_{0}}}\right)$, is a cusp, since $d x_{c} / d \theta=d z_{c} / d \theta=0$, $d^{2} x_{c} / d \theta^{2}=d^{2} z_{c} / d \theta^{2} \neq 0$ for $\theta=\theta_{K}$. The caustic crosses the interface $z=0$ at the point $x_{0 C}=\frac{4}{\alpha} \sqrt{-\alpha z_{0} / 2}$, which is the common point of the branches (4.15a) and (4.15b) for $\theta=\theta_{0 C}=\arctan \left(\sqrt{-\alpha z_{0} / 2}\right)$. It can be easily shown using the parametric equations (4.15a) and (4.15b) that at this point the derived caustic is, in fact, smooth. For initializing Newton's algorithm for solving the system (3.18a), (3.18b), we need to put the caustic equations in the standard form (3.8).

Eliminating $t$ between the parametric equations of the rays (4.12), and putting $y=$ $\cos ^{2} \theta$, we obtain the equations

$$
L(x, z) y^{3}+M(x, z) y^{2}+N(x, z) y+Q(x)=0,
$$

where

$$
\begin{aligned}
L(x, z) & =A(x, z)^{2}+4 \alpha^{2} x^{2}\left(\alpha z_{0}+1\right)^{2}, \\
M(x, z) & =2 A(x, z) B(x, z)-4(\alpha x)^{2}\left(\alpha z_{0}+1\right)\left(\alpha z_{0}+3\right), \\
N(x, z) & =B(x, z)^{2}+4(\alpha x)^{2}\left(3+2 \alpha z_{0}\right), \\
Q(x) & =-4(\alpha x)^{2} \\
A(x, z) & =\left(\alpha z_{0}\right)^{2}-(\alpha z)^{2}+2\left(\alpha z_{0}-\alpha z\right)-(\alpha x)^{2}, \\
B(x, z) & =(\alpha x)^{2}+(\alpha z)^{2}-2\left(\alpha z_{0}-\alpha z\right) .
\end{aligned}
$$




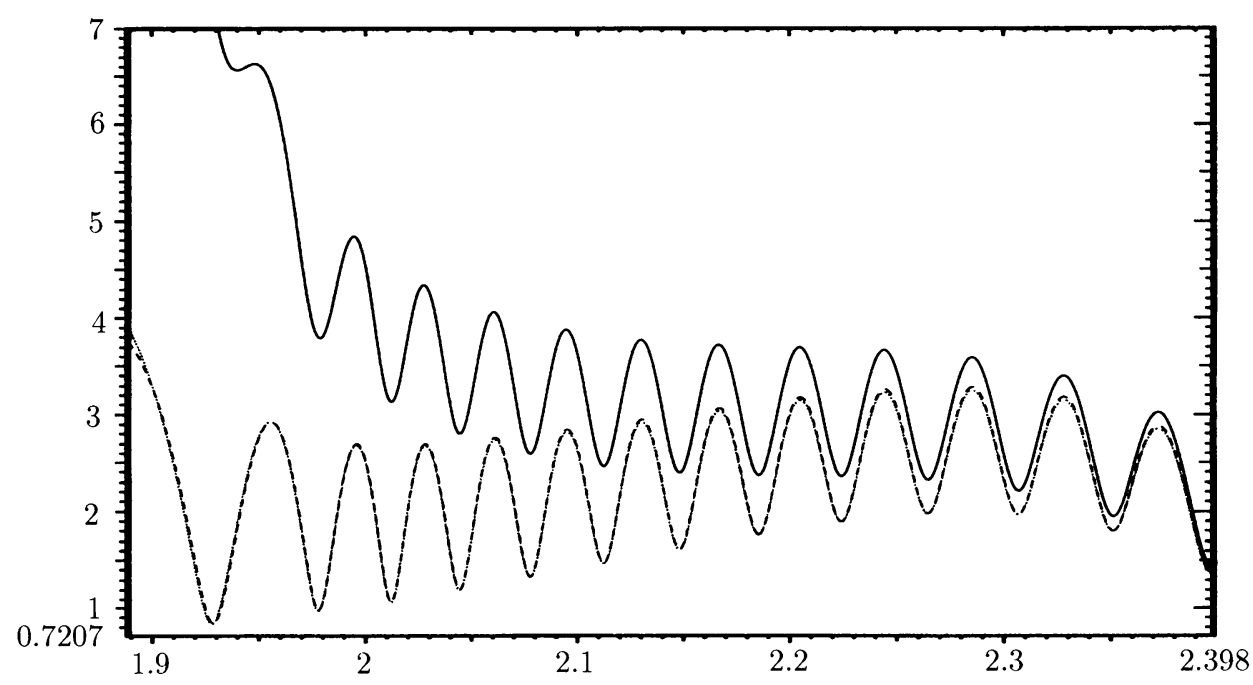

FIG. $4.4 \mathrm{~b} .|u|$ vs. $|x|, \theta=0.44 \mathrm{rad}, k=800$

By the transformation $y=\xi-\frac{M}{3 L}$, we can rewrite (4.17) in the standard form (3.8) of the cusp,

$$
\xi^{3}-\rho_{2} \xi+\rho_{1}=0
$$

where

$$
\begin{aligned}
& \rho_{1}=\frac{1}{L}\left(\frac{2 M^{3}}{27 L^{2}}-\frac{M N}{3 L}+Q\right), \\
& \rho_{2}=-\frac{1}{L}\left(N-\frac{M^{2}}{3 L}\right) .
\end{aligned}
$$

Similarly eliminating $t$ between the equations (4.11) and (4.13), and putting $w=\tan \theta$, we obtain

$$
\begin{gathered}
w+\frac{z_{0}-z}{x}=0, \\
2 w^{2}-\alpha x w-\left(\alpha z+\alpha z_{0}\right)=0 .
\end{gathered}
$$

Multiplying the above equations we find the cubic equation

$$
w^{3}+R(x, z) w^{2}+S w+Q(x, z)=0,
$$

where

$$
\begin{gathered}
R(x, z)=-\frac{\alpha x}{2}+\frac{\left(z_{0}-z\right)}{x}, \\
S=-\alpha z_{0} \\
T(x, z)=\frac{\alpha\left(z^{2}-z_{0}^{2}\right)}{2 x} .
\end{gathered}
$$




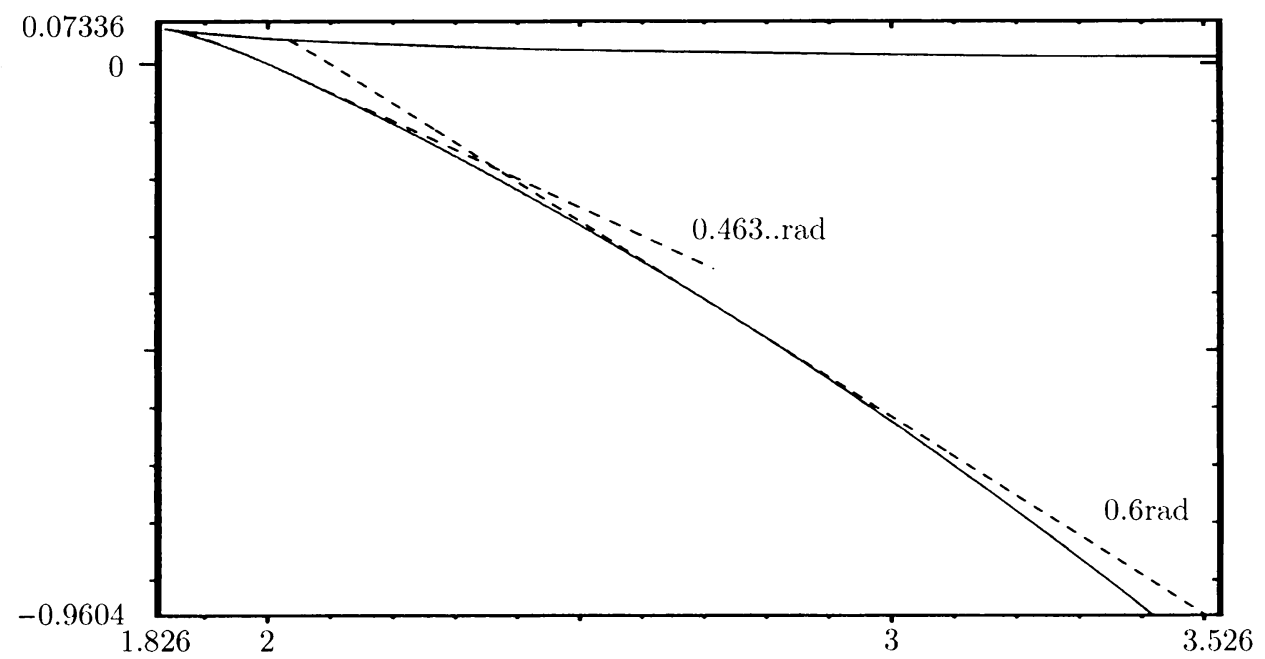

FIG. 4.5. Caustic and rays launched with angle $\theta=\theta_{0 C}=0.463 \mathrm{rad}$ and $\theta=0.6 \mathrm{rad}$

Now, by the transformation $w=\xi-\frac{R}{3}$, we rewrite (4.21) in the form (3.8), choosing

$$
\begin{aligned}
& \rho_{1}=\frac{2 R^{3}}{27}-\frac{S R}{3}+T, \\
& \rho_{2}=\frac{R^{2}}{3}-S .
\end{aligned}
$$

We compare again the GO (continuous line) and KL solutions along some rays for the case $z_{0}=-0.5$ and $\alpha=1$. The ray launched with angle $\theta_{K}=0.38759 \mathrm{rad}$ passes from the beak of the caustic $K$ which has coordinates $\left(x_{K}=1.837117307087383\right.$, $\left.z_{K}=6.0660171779821193 \times 10^{-2}\right)$. The KL solution is computed using both numerical integration (dashed line) and the uniform asymptotic expansion of the Pearcey integral (dotted line). The numerical integration fails by oscillations for certain large values of the arguments in the Pearcey integral. However, it agrees for a satisfactory large range with the numerical integration, to guarantee accuracy of the computation. In Fig. 4.3 we show the caustic and the rays launched from the source with angles $\theta=0.2 \mathrm{rad}$, $0.44 \mathrm{rad}$. The corresponding points of contact between the caustic and the rays are $\left(x=2.687438429694025, z=2.0177387162637617 \times 10^{-2}\right)$ and $(x=1.907681220507820$, $\left.z=3.9775586930300477 \times 10^{-2}\right)$. The computed amplitudes are compared in Figures $4.4 \mathrm{a}$ and $4.4 \mathrm{~b}$. The corresponding wave numbers were selected $k=200$ and $k=800$ to obtain satisfactory accuracy between the numerical integration and the uniform asymptotic expansion used for computing the Pearcey integrals. Similar results for the rays launched with angles $\theta=\theta_{0 C}=0.463 \ldots \mathrm{rad}$ and $\theta=0.6 \mathrm{rad}$ are shown in Figures $4.5,4.6 \mathrm{a}$, and $4.6 \mathrm{~b}$. The points of contact between the rays and the caustic are now $(x=2.0, z=0.0)$ and $(x=2.736547233366769, z=-0.4360863450559146)$, respectively. Note that in Fig. $4.6 \mathrm{~b}$, near $x=2.02$, the GO blows up at the point where the corresponding ray crosses transversely the caustic (see Fig. 4.5). 


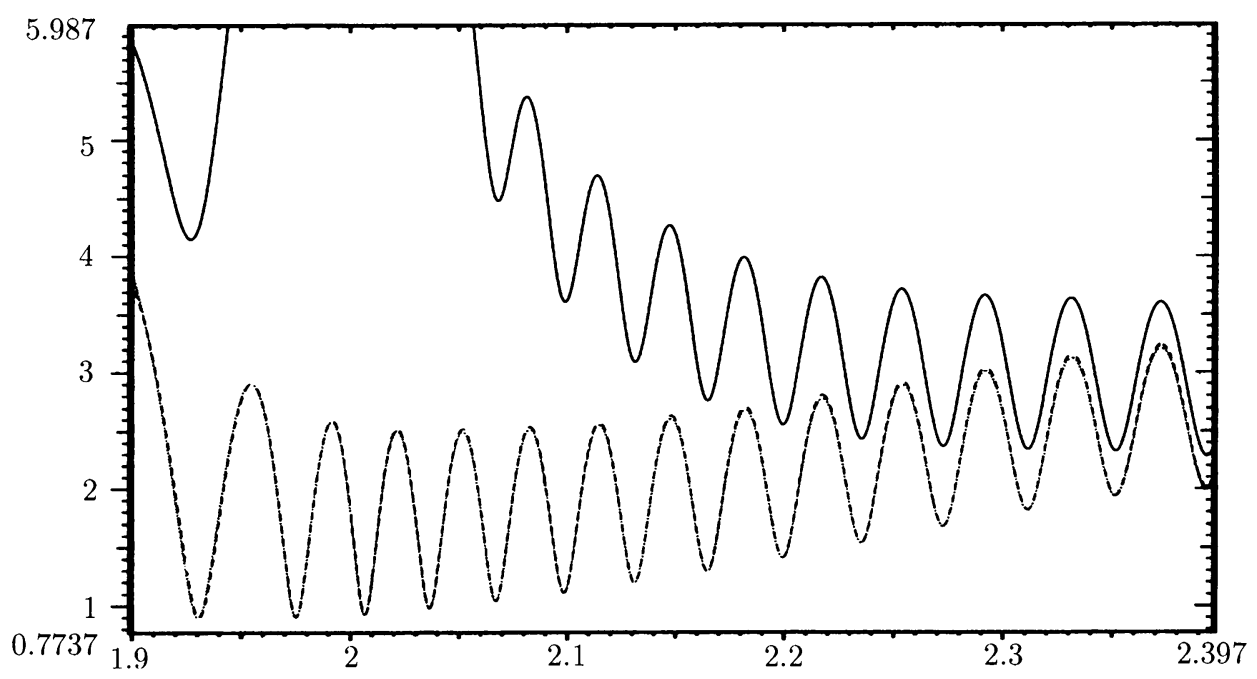

Fig. $4.6 \mathrm{a} .|u|$ vs. $|x|, \theta=\theta_{0 C}, k=800$

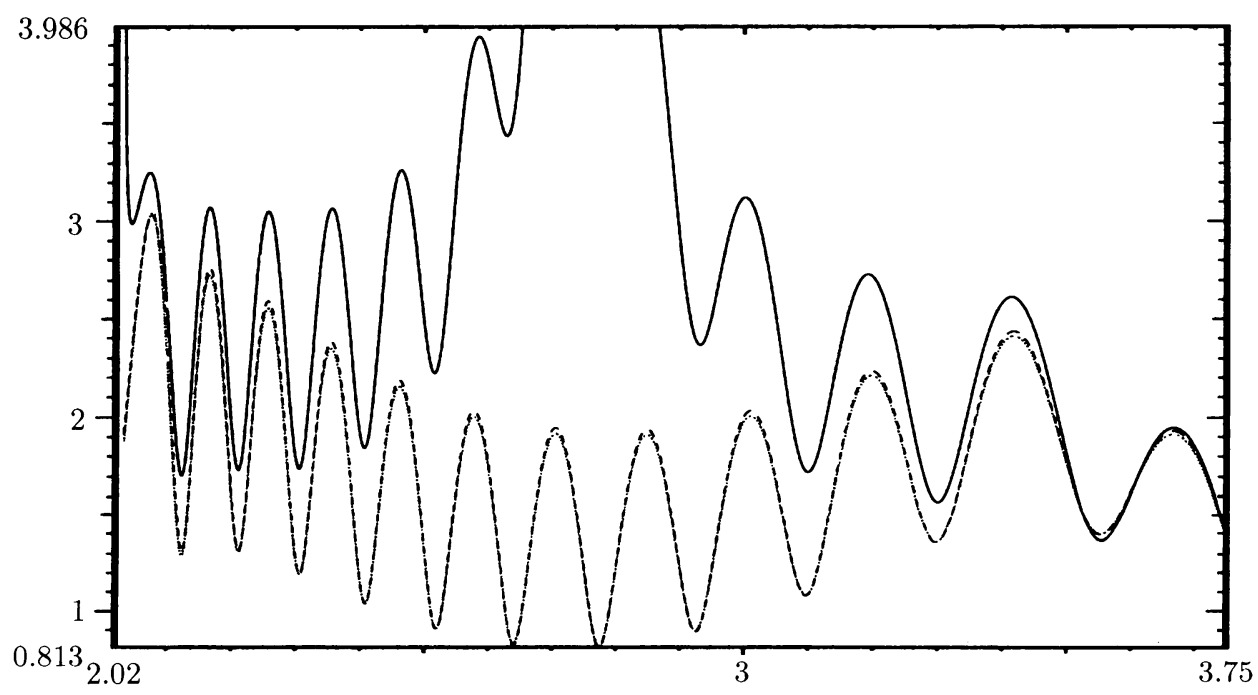

FIG. $4.6 \mathrm{~b} .|u|$ vs. $|x|, \theta=0.6 \mathrm{rad}, k=200$

Acknowledgment. G. N. M. is grateful to Professor Boris Vainberg for his valuable suggestions.

\section{REFERENCES}

[AK] G. Avila and J. Keller, The high-frequency asymptotic field of a point source in an inhomogeneous medium, Comm. Pure Appl. Math. XVI (1963), 363-381.

[ASK] A. A. Asatryan and Yu. A. Kravtsov, Longitudinal caustic scale and boundaries of applicability of uniform Airy-asymptotics, Wave Motion 19 (1994), 1-10. 
[AVH] V. I. Arnold, A. N. Varchenko and S. M. Husein-Zade, Singularities of Differentiable Maps, Vol. 1, Birkhäuser Verlag, Basel, 1985.

[B] V. B. Babich, The short wave asymptotic form of the solution for the problem of a point source in an inhomogeneous medium, USSR J. Comp. Math. and Math. Phys. 5(5) (1965), 949-951.

[BH] N. Bleistein and R. Handelsman, Asymptotic Expansions of Integrals, Dover Publications Inc., New York, 1986.

[BK] R. N. Buchal and J. B. Keller, Boundary layer problems in diffraction theory, Comm. Pure Appl. Math. 13 (1960), 85-144.

[BO] V. A. Borovikov, Uniform stationary phase methods, The Institute of Electrical Engineers, London, 1994.

[BR] L. M. Brekhovskikh, Waves in Layered Media, Academic Press, New York, 1980.

[BT] M. Brown and F. Tappert, Catastrophe theory, caustics and traveltime diagrams in seismology, Geophys. J. R. Astr. Soc. 88 (1987), 217-229.

[CMP] V. Cěrvenỳ, I. A. Molotkov, and I. Pšenčik, Ray Method in Seismology, Univerzita Karlova, Praha, 1977.

[CH1] C. H. Chapman and R. Drummond, Body-wave seismograms in inhomogeneous media using Maslov asymptotic theory, Bull. Seism. Soc. Amer. 72(6) (1982), S277-S317.

[CH2] C. H. Chapman, Ray theory and its extensions: WKBJ and Maslov seismograms, J. Geophys. 58 (1985), 27-43.

[CK] D. Colton and R. Kress, Inverse Acoustic and Electromagnetic Scattering, Springer-Verlag, New York, 1992.

[CF1] J. N. L. Connor and D. Farrelly, Molecular collisions and cusp catastrophes: Three methods for the calculation of Pearcey's integral and its derivatives, Chem. Phys. Let. 81(2) (1981), 306-310.

[CF2] J. N. L. Connor and D. Farrelly, Theory of cusped rainbows in elastic scattering: Uniform semiclassical calculations using Pearcey's integral, J. Chem. Phys. 75(6) (1981), 2831-2846.

[DUI] J. J. Duistermaat, Oscillatory integrals, Lagrangian immersions and unfolding of singularities, Comm. Pure Appl. Math XXVII (1974), 207-281.

[GAR] L. Gárding, Singularities in Linear Wave Propagation, Lecture Notes in Math. 1241, SpringerVerlag, Berlin, 1987.

[GS] V. Guillemin and S. Sternberg, Geometric Asymptotics, Amer. Math. Soc., Providence, R.I., 1977.

[GSC] V. Guillemin and D. Schaeffer, Remarks on a paper of D. Ludwig, Bull. Amer. Math. Soc. 79(2) (1973), 382-385.

[HW] X. Huang and G. West, Effects of weighting functions on Maslov uniform seismograms: A robust weighting method, Bull. Seism. Soc. Amer. 87(1) (1997), 164-173.

[HO] L. Hormander, The Analysis of Linear Partial Differential Operators I, Springer-Verlag, New York, 1983.

[IKM] S. Izumiya, G. T. Kossioris, and G. N. Makrakis, Multivalued solutions to the eikonal equation in stratified media, (submitted).

[KAL] E. Kalligiannaki, Computation of high frequency fields near cusp caustics, Diploma Thesis, Dept. Math., Univ. Crete (Feb. 1999).

[KAM] D. Kaminski, Asymptotic expansion of the Pearcey integral near the caustic, SIAM J. Math. Anal. 20(4) (1989), 987-1005.

[KKM] T. Katsaounis, G. T. Kossioris and G. N. Makrakis, Computation of high frequency fields near caustics, Math. Models Methods in Appl. Sci. 11 (2001), 199-228.

[KO1] Yu. A. Kravtsov and Yu. I. Orlov, Caustics, Catastrophes and Wave Fields, Springer Series on Wave Phenomena, Springer-Verlag, Berlin, 1993.

[KO2] Yu. A. Kravtsov and Yu. I. Orlov, Geometrical Optics of Inhomogeneous Media, Springer Series on Wave Phenomena 6, Springer-Verlag, Berlin, 1990.

[KR] Yu. A. Kravtsov, Two new asymptotic methods in the theory of wave propagation in inhomogeneous media (review), Sov. Phys. Acoust. 14(1) (1968), 1-17.

[KU] V. V. Kucherenko, Quasiclassical asymptotics of a point-source function for the stationary Schrödinger equation, Theoret. Math. Phys. (English Translation) 1(3) (1969), 294-310.

[LE] R. M. Lewis, Asymptotic theory of wave propagation, Arch. Rat. Mech. Anal. 20 (1965), 191-250.

[LEB] N. N. Lebedev, Special Functions and Their Applications, Dover Publications, Inc., New York, 1972. 
[LU] D. Ludwig, Uniform asymptotic expansions at a caustic, Comm. Pure Appl. Math. XIX (1966), $215-250$.

[TC] I. Tolstoy and C. S. Clay, Ocean Acoustics. Theory and Experiment in Underwater Sound, American Institute of Physics, New York, 1966.

[V1] B. R. Vainberg, Quasiclassical approximation in stationary scattering problems, Func. Anal. Appl. 11 (1977), 247-257.

[V2] B. R. Vainberg, Asymptotic Methods in Equations of Mathematical Physics, Gordon and Breach, New York, 1989.

[WED] R. Weder, Spectral and Scattering Theory for Wave Propagation in Perturbed Stratified Media, Springer-Verlag, New York, 1991.

[WO] R. Wong, Asymptotic Approximations of Integrals, Academic Press, Inc., New York, 1989.

[ZD] R. W. Ziolkowski and G. A. Deschamps, Asymptotic evaluation of high-frequency fields near a caustic: An introduction to Maslov's method, Radio Sci. 19(4) (1984), 1001-1025. 